\title{
Adrenal Cortex Carcinoma: One of the Rarest Retroperitoneal Tumors in children
}

ISSN: 2576-9200

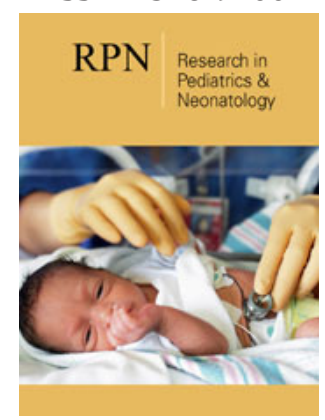

*Corresponding author: D Hanine, Pediatric Surgery Department "A", Children's Hospital of Rabat, Faculty of Medicine and Pharmacy, Mohammed V University Rabat, Morocco

Submission: 誨 April 01, 2021

Published: 制April 29, 2021

Volume 5 - Issue 4

How to cite this article: $D$ Hanine, $B$ Rouijel, A El Baoudi, M Kisra. Adrenal Cortex Carcinoma: One of the Rarest Retroperitoneal Tumors in children. Research in Pediatrics \& Neonatology. 5(4). RPN. 000618. 2021.

DOI: 10.31031/RPN.2021.05.000618

Copyright $@$ D Hanine. This article is distributed under the terms of the Creative Commons Attribution 4.0 International License, which permits unrestricted use and redistribution provided that the original author and source are credited.

\author{
D Hanine ${ }^{1,2 *}$, B Rouijel ${ }^{1,2}$, A El Baoudi ${ }^{1,2}$ and M Kisra ${ }^{1,2}$ \\ ${ }^{1}$ Pediatric Surgery Department “A", Children's Hospital of Rabat, Morocco \\ ${ }^{2}$ Faculty of Medicine and Pharmacy, Mohammed V University Rabat, Morocco
}

\begin{abstract}
Adrenal cortex is a cancer of the adrenal cortex, rare in children; it accounts for $0.2 \%$ of pediatric cancers with a ratio of 1.5 in girls and is sometimes part of predisposing syndromes. The clinical signs suggestive of adrenocortical carcinoma are most frequently endocrine, present in $90 \%$ of cases. The hormonal increased secretion is made up of cortisol, androgens, estrogen and more rarely, Aldosterone. Symptoms of virilization are most commonly seen and may or may not be associated with Cushing's syndrome. Arterial hypertension (hypertension) caused by increased secretion of aldosterone is rarer. More rarely, the mode of discovery is the presence of an abdominal mass, most often associated with abdominal pain, or sometimes local signs of compression.

The prognosis of these tumors, as well as the survival rate, is very variable depending on the tumor stage. It is very pejorative for stage III and IV tumors, compared to stage I and II tumors. The 5-year overall survival rate, all stages combined, fluctuates between $49 \%$ and $57 \%$ depending on the series, but it can vary between 20 and $90 \%$ depending on the tumor stages. Surgery is the cornerstone of treatment, hence the question all surgeons ask themselves: the possibility of complete excision. We report the case of an infant, brought to consult for a Genu Varum, who's clinical and radiological investigations concluded in an adrenal cortex. The treatment was surgical with an anatomopathological study confirming the diagnosis. The outcome was favorable thereafter with regression of endocrine signs over a 6-month follow-up. At the end of this observation, we highlight this very rare pathology as well as its management.
\end{abstract}

Keywords: Adrenocortical carcinoma; Virilizing tumors; Adrenal tumors; Child

\section{Introduction}

Adrenocortical Carcinoma (ACC) are rare tumors that have a bimodal distribution, the first peak is in children less than five years and the second around the fifth decade [1]. Although most adult ACC are non-functional, in the pediatric age group, nearly $95 \%$ are functional [2]. Virilization is the most common abnormality and Cushing's syndrome and hyperaldosteronism are less frequent [3]. We begin the review with a clinical case of a 16-month-old infant, and then we will discuss this very rare pathology as well as its management.

\section{Materials \& Methods}

Our study was done in March 2021 at the pediatric surgery department "A" at the Children's Hospital of Rabat, the case studied is a 16-month-old boy brought by his parents for hirsutism and genu varum. Without history, the child had since the first months a pubic hair. On examination, his weight was $12 \mathrm{~kg}$, height was $85 \mathrm{~cm}$ and blood pressure was $90 / 60 \mathrm{mmHg}$. He had no cushingoid face, no moon face, not stretch marks, but pubic and body hair well developed and gynecomastia. The external genitalia were male with normal sized testicles but an increased penis of $6 \mathrm{~cm}$ in size. On the osteoarticular level, the articular amplitudes were normal. The clinical examination showed a harmonious bilateral genu varum. There were no neurocutaneous markers. There was no family history of malignancy. Laboratory investigations revealed normal hematological and biochemical parameters. Morning $(0800$ hours) serum cortisol was $2.9 \mu \mathrm{g} / \mathrm{Dl}$. Other hormonal levels were normal.

Ultrasound of abdomen revealed a tissue mass at the level of the superior pole of the right kidney, heterogeneous hypoechoic, of regular contours, exerting a mass effect on the renal cortex without separating border; measuring 52x51x44mm. Computed Tomography (CT) of 
the abdomen showed a right retroperitoneal mass, well-defined tissue, of regular contours, without calcifications, presenting a heterogeneous enhancement after injection of the contrast product; measuring $51 \times 43 \times 49 \mathrm{~mm}$ (Figure 1 ). The extension assessment was negative. We decide to do a first surgery. Surgical exploration found a fully encapsulated right adrenal mass. This mass pushed back the inferior vena cava and the renal pedicle without infiltrating them with a separating border. The dissection of the mass above the liver was easy. We continued dissection down below the Kidney (Figure 2). We did a complete resection of the encapsulate mass (Figure 3). There was no locoregional ganglion. The anatomopathological study concluded in an Adrenocortical Carcinoma (ACC). The postoperative follow-up was simple with regression of signs of virilization in the postoperative months.

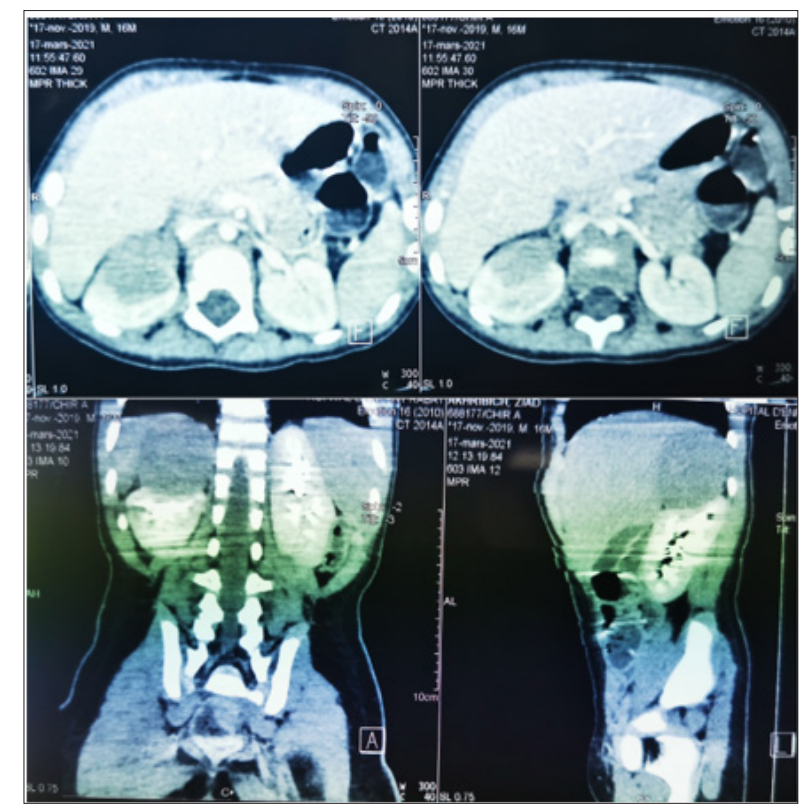

Figure 1: Abdominal CT showing the tumor.

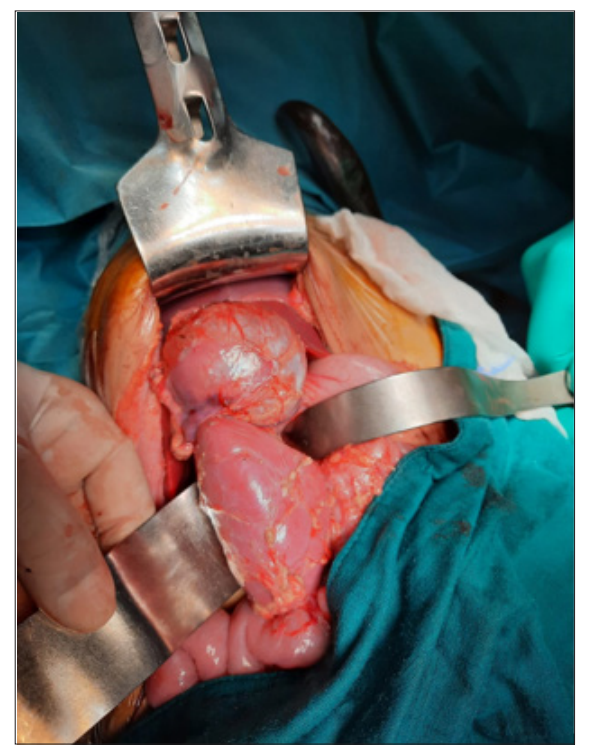

Figure 2: Surgical exploration showing the mass at the upper pole of the Kidney.

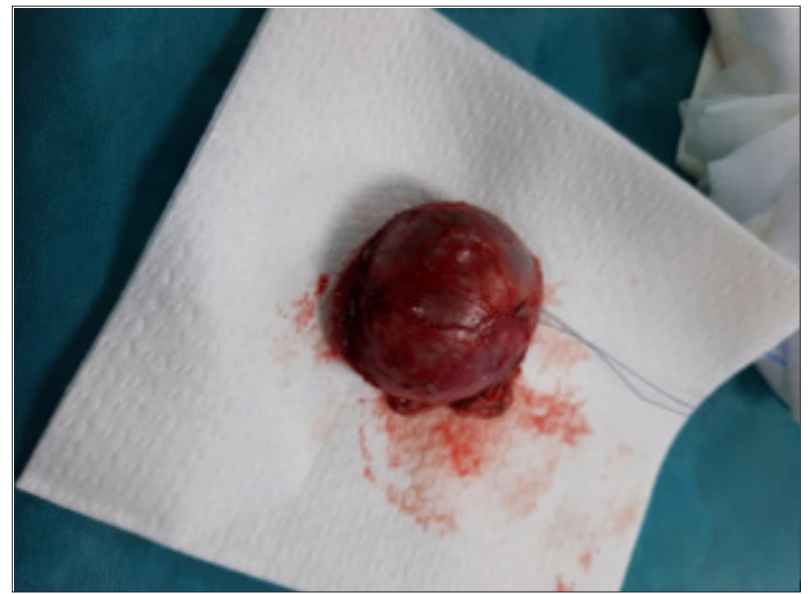

Figure 3: The fully encapsulated resected mass.

\section{Discussion}

Adrenal cortex in children is rare, accounting for only $0.2 \%$ of pediatric cancers. The incidence of ACC follows a bimodal distribution with a peak during the first ten years of life and particularly before four years. It is estimated at $0.4 /$ million per year for the first four years of life [4]. The ACC can be on the right or left side, however some authors recognize a greater frequency of these tumors on the left side. Bilateral tumors have been reported in $2-10 \%$ of cases [5]. The sex ratio changes with the age at the time of diagnosis of the disease. There is an overall female predominance, accentuating among those under three (ratio: 1.7/1) and especially among those over 13 (ratio: 6.2/1) [2]. ACCs can occur as part of specific predisposing syndromes that are currently well identified, the two main ones being: [6].

\section{Li Fraumeni syndrome}

Li Fraumeni syndrome is an autosomal disease characterized by a predisposition and early onset of several cancers; this syndrome should be looked for systematically in children with ACC.

\section{Beckwith-wiedemann syndrome}

Beckwith-Wiedemann Syndrome is characterized by visceralomegaly, hyperglyceridemia, malformations, and predisposition to malignant tumors.

\section{Other predisposing syndromes}

A. NEM1: multiple endocrine neoplasia 1: rarely involved in children.

B. Carney complex: mainly responsible for lumpy disease of the adrenal cortex.

C. Congenital adrenal hyperplasia most often responsible for adenomas.

In children, the majority of ACCs are of the secreting type while only less than $50 \%$ of these tumors appear functional in adults. In more than $90 \%$ of cases the clinical signs suggestive of ACC are most frequently endocrine, most often made of a syndrome of virilization associated or not with Cushing syndrome, feminization syndrome or Conn syndrome are rare [7]. 
Virilizing tumors are the most frequent presentation of the childhood ACC, it largely predominates in the series studied: 95\% for SABBAGA [8], 76\% for C TEINTURIER [9], 66\% for Sprague RG [5], 55\% for Sandrini R [7]. These tumors are manifested by iso-sexual precocious pseudo-puberty in boys and heterosexual in girls with a picture of virilization. Most often the first sign that attracts attention is an enlarged clitoris in girls or the penis in boys. Sometimes attention is drawn to the appearance of pubic hair, accelerated growth, change in voice [7].

In boys: The picture is that of precocious pseudo-puberty with hypertrophy of the penis which is constant, the length of the penis is variable ranging from 4 to $10 \mathrm{~cm}$, our patient had a penis of $5 \mathrm{~cm}$; the testicular volume remains normal for the age[10]. This points to adrenal pathology, the presence of pubic hair is constant in both sexes to varying degrees, it is not proportional to the degree of hypertrophy of the clitoris or the penis [11]. Facial and axillary hairs are also reported in some observations.

Regarding the change in growth: The excess of androgens leads to an acceleration of height and weight growth and bone maturation most often the height of children is greater than the normal average for their age bone maturation is accelerated, bone age is advanced compared to chronological age [11]. The excess weight is also noted, it is due to the muscular hypertrophy which accompanies virilism. The acceleration of bone maturation causes premature growth arrest by early closure of the epiphyses of the long bones.

\section{Hypertension}

This hypertension is explained by some authors by compression of the renal vessels by a large tumor [7]. Some observations show that HTA is present even in the case of a small tumor, this can be explained by the tumor secreting mineralocorticoids with a hypertensive effect [12].

\section{In infants}

Obesity: this is the first sign that sets in, it is most often a generalized obesity with a moon face, a bison hump, and rarely, it is similar to Cushingoid obesity of the adult. Arterial hypertension: for SABBAGA the hypertension is almost constant $90 \%$ of cases while C TEINTURIER only found it in $30 \%$ of cases [13]. Other signs are muscle deficit, diabetes and osteoporosis (less common than in adults), hirsutism. Cutaneous (acne, stretch marks) and neuropsychological signs are rare [14].

Feminizing tumors: 5.6. Feminizing tumors are rare $[12,15,16]$. Conn Syndrome is very rare, few cases have been reported in the literature, hypermineralocorticism is the cause [17]. Regarding non-secreting tumors, they represent only 5\% of childhood ACCs. They have a poor prognosis because at the time of diagnosis, the disease is often advanced. The clinical signs of these tumors are linked [18], on the one hand, to the mechanical phenomena generated by the invasion of neighboring structures; on the other hand, to metastatic swarming. The diagnosis can be made before abdominal pain, abdominal mass, weight loss. Other signs can be found digestive signs, general signs: anorexia, fever, asthenia, hypertension of renal origin, inferior vena cava syndrome, Budd Chiari syndrome.

The presence of metastases at diagnosis is uncommon in children at $13 \%$, unlike metastases in adults, which is 22 to $50 \%$. The standard non-hormonal biological workup may reveal nonspecific abnormalities: an inconstant inflammatory syndrome. Concerning hormonal biological signs, the biological diagnosis of Cushing syndrome is made by the determination of serum and urinary cortisol [19-21]. The assay of total testosterone is recommended as a first-line treatment, the assay method chosen must be specific and sensitive to assay low concentrations. DHEA and SDHEA are the most specific. $90 \%$ of children with virilizing ACC have a high plasma SDHEA level [7]. The dosage of urinary 17 ketosteroids is particularly interesting since it is high in almost all patients with virilization syndrome [7]. Do not forget to eliminate a pheochromocytoma by assaying plasma meta and normetanephrines and urinary catecholamines. These hormonal assays therefore make it possible to guide the diagnosis. It is important to perform them before any treatment and in particular before any surgical procedure, because their iterative performance after treatment can make it possible to follow the evolution of the disease and to detect early tumor recurrence in the event of reascension after Initial normalization.

Radiologically; ultrasound cannot reliably detect even the smallest adrenal lesions [21,22]. Thoraco-abdominopelvic Computed Tomography (CT) is the gold standard [23,24]. ACC appears on CT as a heterogeneous mass, with irregular margins and a spontaneous density> $10 \mathrm{HU}$, the size of which exceeds $5 \mathrm{~cm}$. It can present numerous calcifications, very often with necrotic or hemorrhagic areas. This tumor enhances heterogeneously after injection of the contrast product (42\%). It presents a strong venous tropism with possible extension to the renal vein or the inferior vena cava. This examination also makes it possible to assess the presence of a locoregional invasion (adipose tissue, mediastinalabdominal and pelvic lymph nodes, vena cava, renal vein, adjacent organs) or distant metastases (liver, lung, bone).

MRI can also complement abdominal CT data to refine the assessment of locoregional, metastatic, vascular or lymph node extension. It makes it possible to better define the relationships with neighboring organs, in particular on the right with the liver and above all it shows with precision the renal veins and the IVC up to the right atrium and detects a tumor extension, with an almost anatomical image, allowing true pre-surgical mapping [25]. On MRI, the lesion appears hypointense in T1, hyperintense in T2; sometimes there are very limited areas of intracytoplasmic fat. On the out-of-phase sequences, an increase in the signal is obtained. The extension workup is done with brain CT and bone scintigraphy.

Pathological analysis remains the cornerstone of the diagnosis of adrenal tumors and in particular ACC. However, it remains a real challenge, including for the most experienced pathologists for two main reasons. The first difficulty is to establish with certainty the 
origin of the adrenal cortex of the lesion. For this, immunostaining of Steroidogenic Factor 1 (SF-1) appears to be the most sensitive and specific marker. The second difficulty is to discriminate the benignity and the malignancy of the lesion [23]. The potential for malignancy of an adrenal cortex tumor remains difficult to establish, particularly in children. A different histopronostic score than the adult Weiss score has been proposed by the Armed Forces Institute Of Pathology (AFIP) for childhood tumors without being completely reliable [26]. The classification of childhood ACC was proposed by Sandrine and his team and modified from data from the international registry and distinguishes 4 stages [27].

Preoperative preparation is essential in patients with hypercriticism, tumor secretion leads to suppression and atrophy of the contralateral gland; once the tumor is resected, adrenal insufficiency sets in [28]. Surgery is the key, since "complete" removal of a localized form, intra-adrenal tumor (stage II) or slightly advanced (certain stages III), is the best chance of a real cure. The presence of intraoperative tumor rupture increases the risk of locoregional recurrence according to Sandrini and Michalkiewicz. Ruptured tumors are therefore now considered stage III and adjuvant therapy is currently recommended in these patients. This rupture can also be spontaneous and be responsible for an acute abdomen, or follow a needle biopsy performed for diagnostic purposes. This gesture is therefore to be avoided. There is little data in the literature on the incidence and role of lymph node invasion in children because there are no clear recommendations for taking lymph node samples during surgery. Based on experience at ST. JUDE CHILDREN'S RESEARCH HOSPITAL, the presence of lymph node invasion is associated with a poor prognosis.

The recommendation is to take lymph node samples at the time of resection of the primary tumor and to perform lymph node dissection when the result of the biopsy is positive [29]. The role of chemotherapy is not yet clearly established in the management of childhood ACC. The response rate varies between 7 and 54\%, with great variability in the response criteria [30]. Progress made in the field of genomic analysis techniques and better knowledge of the molecular players involved in the pathogenesis of ACC in adults but also in children has made it possible to consider the use of so-called targeted therapies. Faced with the absence of truly discriminating anatomopathological prognostic markers, many studies have shown the importance of clinical prognostic factors, such as tumor size and weight. However, the thresholds set vary from study to study.

Complete tumor resection is the most important prognostic factor. Long-term survival is approximately $75 \%$ in children who have had the tumor completely removed. Patients who have a microscopic or macroscopic residue after surgery have a poor prognosis [31]. Another factor is the type of tumor secretion: children whose ACC secrete excess glucocorticoids seem to have a poor prognosis, unlike those with pure signs of virilization [31]. The age at diagnosis appears to be an important prognostic factor. Children under 2 years of age have a long-term survival rate of $82 \%$ [8]. In contrast, children over 2 years of age have a survival rate of $29 \%$ [32]. The five-year overall survival rate of children with ACC ranges from 54 to $74 \%$ depending on the series.

\section{Conclusion}

Adrenal Cortex Carcinoma (ACC) is a malignant tumor of the adrenal cortex that most commonly occurs in adulthood between the ages of 40 and 50 . These tumors are extremely rare in children, corresponding to only $0.2 \%$ of childhood cancers. The sex ratio is in favor of girls. There are predisposing genetic factors in almost $50 \%$ of cases. The main mode of discovery of a SCC is the presence of endocrine signs by hormonal hypersecretion found ifn $80 \%$ to $90 \%$ of cases. Symptoms of virilization are most frequently observed. The diagnosis is based on clinical and paraclinical investigations and confirmed by pathological examination. The standard treatment for SCC is surgical excision, which should be complete if possible. When this resection is impossible or incomplete, and in metastatic forms, recourse to adjuvant or alternative treatment may be necessary. The prognosis of these tumors, as well as the survival rate, is very variable depending on the tumor stage.

\section{References}

1. Ng L, Libertino JM (2003) Adrenocortical carcinoma: Diagnosis, evaluation and treatment. J Urol 169(1): 5-11.

2. Michalkiewcz E, Sandrini B, Figueiredo B, Miranda EC, Caran E, et al. (2004) Clinical and outcome characteristics of children with adrenocortical tumors: A report from the international pediatric adrenocortical tumor registry. J Clin Oncol 22(5): 838-845.

3. Cagle PT, Hough AJ, Pysher TJ, Johnson EH, Kirkland RT, et al. (1986) Comparison of adrenal cortical tumors in children and adults. Cancer 57: 2235-2237.

4. Ribeiro RC, Figueiredo B (2004) Childhood adrenocortical carcinoma. Eur J Cancer 40(8): 1117-1126.

5. Liou LS, Kay R (2000) Adrenocortical carcinomain children review and recent innovations. Urol Clin North Am 27(3): 403421.

6. Espiard S, Bertherat J (2015) The genetics of adrenocortical tumors. Endocrinol Metab Clin North Am 44(2): 311-334.

7. Leblond P, Delebarre M, Aubert S (2011) Management of adrenocortical carcinomas in children. Bull Cancer 98(5): 595605.

8. Sabbaga CC, Avilla SG, Schulz C, Garbers JC, Blucher D (1993) Adrenocortical carcinoma in children: Clinical aspects and prognosis 28(6): 841-843.

9. Teinturier C, Brugières L, Lemerle J, Chaussain JL, Bougnéres PF (2000) Child adrenal cortex retrospective analysis of 54 cases.

10. Kumar S, Tiwari P, Kr Das R, Kr Kundu A (2010) Virilizing adrenal carcinoma in a 3-year-old boy: A rarity. Indian J Med Paediatr Oncol 31(1): 30-32.

11. Burrington JD, Stephens CA (1969) Virilizing tumors of the adrenal gland in childhood: Report of eight cases. J Pediatr Surg 4(3): 291-302.

12. Bouyahia O, Gharsallah L, Ouederni M, Boukthir S, Mrad SM, et al. (2009) Feminizing Adrenocortical Adenoma in a 5 Year-old Girl. J Pediatr Endocrinol Metab 22(1):79-84. 
13. Elisabethe B Fudge, Daniel L von Allmen (2009) Cushing syndrome in a 6-month-old infant due to adrenocortical tumor. Int J Pediatr Endocrinol 2009: 168749.

14. Stratakis CA (2012) Cushing Syndrome in Pediatrics. Endocrinol Metab Clin North Am 41(4): 793-803.

15. Ghazi AA, Mofid D, Rahimi F, Marandi H, Nasri H, et al. (1994) Oestrogen and cortisol producing adrenal tumour. Arch Dis Child 71(4): 358-359.

16. Arnao JR, Perry L, Dacie JE, Reznek R, Ross RJ (1995) Primary hyperaldosteronism due to an adrenal adenoma in a 14-year-old boy. Postgrad Med J 71(832): 104-106.

17. Bremont C, Luton IP (1998) Cushing syndromes. The practitioner's review, Paris monograph tome 48: 738-743.

18. Libe R, Assie G (2014) Adrenal cortex: New in 2014.

19. Plouin PF, Simon CM (1998) Primary hyperaldosteronism. Rev Prat 48(7): 749-753.

20. Artus PM, Miquel C, Meria P, Hernigon A, Duclos JM (2004) Secreting adrenocortical tumors, Adrenocortical secretory tumors. Annals of Urology 38(4): 148-172.

21. Rakoto-Ratsimba HN, Razafimahandry HJC, Ravalisoa A, Ranaivozanany A (2003) A case of giant malignant adrenocortical carcinoma. Ann Urol (Paris) 37(1): 17-20.

22. Jebbari A, Nassar I, Edderai M, Bouklata S, Hammani L, et al. Principles of analysis of an adrenal mass: Contribution of CT and MRI. Radiology Department, Ibn Sina University Hospital, Rabat, Morocco.

23. Oddoze C, Castinetti F (2013) Adrenal cortical pathologies and exploration of glucocorticoids 12(314).

24. Benchekroun, Ghadouane, Alami M, Kassmaoui EH, Benslimane L, et al. (2000) Malignant adrenocortical carcinoma: About 22 cases. Advances in Urology 10: 205-210.
25. (2010) Imageries of the adrenals. College of teachers of endocrinology, Diabetes and Diseases. Metabolics (CEEDMM).

26. Wieneke JA, Thompson LDR, Heffess CS (2003) Adrenal cortical neoplasms in the pediatric population: A clinicopathologic and immunophenotypic analysis of 83 patients. Am J Surg Pathol 27(7): 867-881.

27. Ribeiro RC, Pinto EM, Zambetti GP, Rodriguez-Galindo C (2010) The international pediatric adrenocortical tumor registry initiative: contributions to clinical, biological, and treatment advances in pediatric adrenocortical tumors. Mol Cell Endocrinol 351(1): 37-43.

28. Zeiger MA, Thompson GB, Duh QY, Hamrahian AH, Angelos P, et al. (2009) American association of clinical endocrinologists and american association of endocrine surgeons medical guidelines for the management of adrenal incidentalomas: Executive summary of recommendations. Endocr Pract 15(5): 450-453.

29. Stewart JN, Flageole H, Kavan P (2004) A Surgical approach to adrenocortical tumors in children: The mainstay of treatment. Journal of Pediatric Surgery 39(5): 759-763.

30. Baudin E, Leboulleux S, Al Ghuzlan A, Chougnet C, Young J, et al. Therapeutic management of advanced adre- nocortical carcinoma: what do we know in 2011? Horm Cancer 2(6): 361371

31. Ribeiro RC, Pinto EM, Zambetti GP (2010) Familial predisposition to adrenocortical tumors: Clinical and biological features and management strategies. Best Pract Res Clin Endocrinol Metab 24(2010): 477-490.

32. Ciftci AO, Senocak ME, Tanyel FC, Büyükpamukçu N (2001) Adrenocortical tumors in children. J Pediatr Surg 36(4): 549554. 